\title{
A COMPARISON OF THE PREDICTIVE ABILITY OF POOLED COHORT EQUATION, SCORE EQUATION AND D:A:D STUDY EQUATION IN PEOPLE LIVING WITH HIV
}

M. Papagianni ${ }^{1}$, A. Evangeliou ${ }^{1}$, S. Mamalis ${ }^{1}$, E. Karypidou ${ }^{1}$, O. Tsachouridou ${ }^{1}$, A. Georgiou ${ }^{1}$, C. Gogou $^{1}$, T. Chrysanthidis ${ }^{1}$, D. Valagouti ${ }^{1}$, P. Kollaras ${ }^{1}$, K. Tziomalos ${ }^{2}$, P. Zembekakis ${ }^{3}$, S. Metallidis ${ }^{1}$.

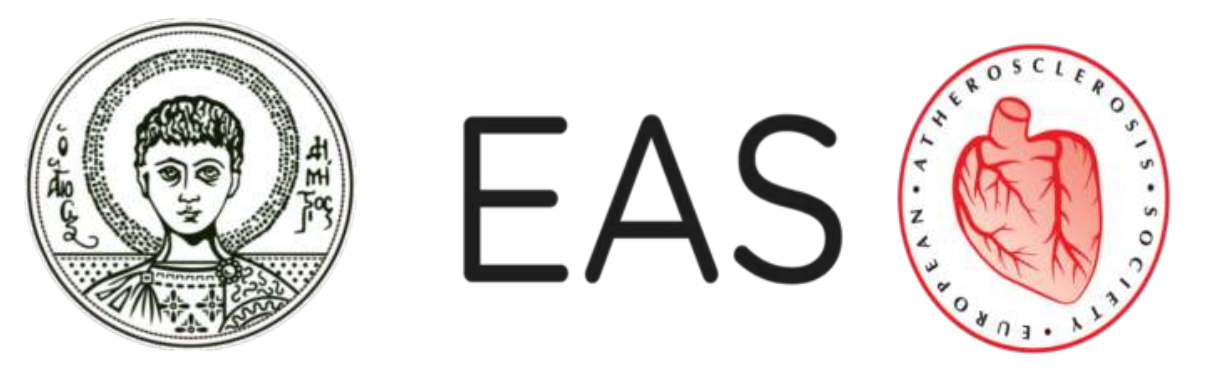

IInfectious Disease Department- First Department of Internal Medicine- AHEPA Hospital, Medical School- Aristotle University of Thessaloniki, Thessaloniki, Greece.

${ }^{2}$ First Propedeutic Department of Internal Medicine- AHEPA Hospital, Medical SchoolAristotle University of Thessaloniki, Thessaloniki, Greece.

${ }^{3}$ First Department of Internal Medicine- AHEPA Hospital, Medical School- Aristotle University of Thessaloniki, Thessaloniki, Greece.

BACKGROUND AND AIMS

The aim of the study is to compare the predictive ability of the American College of Cardiology/American Heart Association Pooled Cohort equations, the European Society of Cardiology Systematic Coronary Risk Evaluation (SCORE) equation and the Data Collection on Adverse Effects of AntiHIV Drugs (D:A:D) study equation in persons living with human immunodeficiency virus (HIV).

\section{METHODS}

We retrospectively studied all patients with HIV infection who had at least one clinical encounter in 2008 and at that time were 40-65 years-old and did not have established cardiovascular disease or diabetes. The cardiovascular risk was estimated with the Pooled Cohort equations, the SCORE equation and the D:A:D study equation. Patients were followed-up for 10 years.

\section{RESULTS}

In 2008, 232 patients aged 40-65 years old, without diabetes or prior cardiovascular disease were enrolled in our study (76.3\% males, mean age $47.1 \pm 6.6$ years). The predicted 10 -year risk for total CV events with the Pooled Cohort equation was $6.5 \pm 4.6 \%$ whereas the observed risk was $7.8 \%$. The predicted 10 -year risk for fatal CV events with the SCORE equation was $2.5 \pm 2.5 \%$ whereas the observed risk was $0.9 \%$. The predicted 5-year risk for total CV events was 3.5 $\pm 3.0 \%$ with the D:A:D study equation whereas the observed 5-year risk was $6.5 \%$.

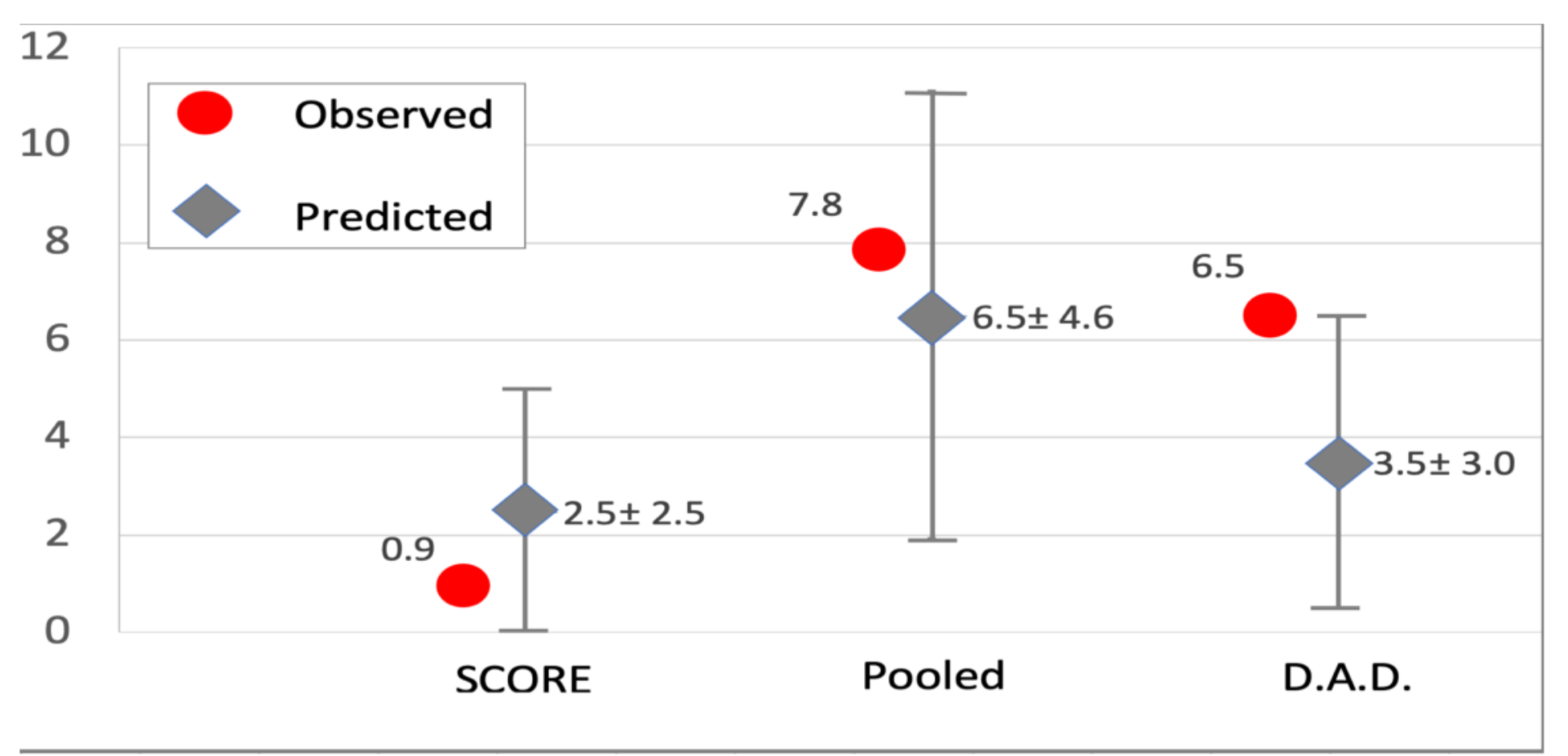

\begin{tabular}{cccc}
\hline & $\begin{array}{c}\text { Patients who } \\
\text { suffered a CV } \\
\text { event }(\mathrm{n}=18)\end{array}$ & $\begin{array}{c}\text { Patients who did not } \\
\text { suffer a CV event }\end{array}$ & P-value \\
& $49.4 \pm 9.3$ & $40.1 \pm 10.0$ & $<0.001$ \\
\hline Age (y) & 45.5 & 15.6 & $<0.001$ \\
HTN (\%) & $96 \pm 25$ & $109 \pm 25$ & $<0.05$ \\
GFR (ml/min/1.73m2) & $14,75 \pm 6,1$ & $8,58 \pm 5,58$ & $<0.001$ \\
NRTIs exposure (y) & \multicolumn{3}{l}{ y: years, HTN: Hypertension, GFR: Glomerular filtration rate, CV: Cardiovascular } \\
\hline
\end{tabular}

\begin{tabular}{lc}
\hline \multicolumn{2}{c}{ Patients Characteristics } \\
\hline Age (years) & $47.1 \pm 6.6$ \\
Males (\%) & 76.3 \\
\hline Intravenous drug use (\%) & 8.6 \\
Family history of CVD (\%) & 4.7 \\
Hypertension (\%) & 25.9 \\
Package-years & $20.5 \pm 17.8$ \\
\hline Body mass index (kg/m²) & $25.4 \pm 3.9$ \\
\hline Total cholesterol (mg/dl) & $210 \pm 49$ \\
LDL cholesterol (mg/dl) & $126 \pm 41$ \\
\hline HDL cholesterol (mg/dl) & $46 \pm 16$ \\
Triglycerides (mg/dl) & $194 \pm 120$ \\
Glomerular filtration rate (ml/min/1.73m²) & $105 \pm 25$ \\
Chronic kidney disease (\%) & 2.6 \\
\hline Systolic blood pressure (mmHg) & $126 \pm 14$ \\
Diastolic blood pressure (mmHg) & $81 \pm 10$ \\
Duration of HIV infection (years) & $7.2 \pm 5.6$ \\
Antiretroviral treatment (\%) & 80.2 \\
\hline
\end{tabular}

\section{CONCLUSIONS}

The SCORE showed excellent discrimination whereas the Pooled and the D:A:D study equation showed acceptable discrimination (Cstatistic $0.85,0.75$ and 0.76, respectively; Table 3). The Pooled and the D:A:D study equation also showed good calibration (HosmerLemeshow $\chi 2$ value 3.26 and 10.52, respectively (on 8 degrees of freedom); $p=0.92$ and $p=0.23$, respectively). In contrast, the SCORE was not well calibrated (Hosmer-Lemeshow $\chi 2$ value 13.60 (on 4 degrees of freedom), $p=0.009$ ). Overall, 3.0, 33.2 and 3.9\% of the study population was classified as very high cardiovascular risk according to the SCORE, the Pooled and the D:A:D study equation. The Pooled showed poor agreement with both the SCORE and the D:A:D study equation (Cohen's kappa coefficient 0.118 and 0.125 , respectively). In contrast, the SCORE showed better agreement with the D:A:D study equation (Cohen's kappa coefficient 0.353). 